\title{
Factores clave para el proceso de gestión de la innovación para el desarrollo de nuevos productos en el sector PyMEs, caso de estudio la industria de tecnologías de la información en Nuevo León \\ (Key factors for the process innovation management for new product development in the SME sector, case study information technology in Nuevo León)
}

\author{
Óscar Enrique López Treviño \& Mónica Blanco Jiménez \\ UANL, San Nicolás de los Garza, N.L., México, lopezoscarenrique@yahoo.com.mx
}

Key Words. canieti, innovation management process, new product development (NPD), organizational creativity, SMEs.

\begin{abstract}
The purpose of this study is to analyze the key factors for managing the product innovation process, from existing models over time. Different proposals are presented by different authors who have studied this phenomenon, and as a result of these have conceptualized the knowledge in different models of innovation, so this work focuses on meeting certain elements in a new model including a new variable was not been considered in previous models (Organizational Creativity). This study was focused on SMEs and their importance in the economy of any country. The analysis of results and findings are presented from a study of 53 samples to the industry of information technology in Nuevo León, México.
\end{abstract}

Palabras Clave. Canieti, desarrollo de nuevos productos (DNP), proceso de gestión de la innovación, PyMEs.

Resumen. El propósito del estudio es analizar los factores clave para gestionar el proceso de innovación de producto, a partir de modelos existentes a través del tiempo. Se presentan diferentes propuestas por distintos autores que han estudiado este fenómeno, y como resultado de estos han conceptualizado ese conocimiento en diferentes modelos de innovación, por lo que este trabajo se enfoca en conjuntar ciertos elementos en un modelo nuevo incluyendo una variable nueva que no había sido considerada en modelos anteriores (Creatividad Organizacional). Este estudio va enfocado a las PyMEs por la importancia que tienen en la economía de cualquier país. El análisis de resultados y las conclusiones se presentan a partir de un estudio de 53 muestras a la industria de las tecnologías de la información en Nuevo León, México. 


\section{Introducción}

La investigación científica que se propone en el siguiente documento se basa en la tarea de seleccionar, desarrollar y probar una propuesta de valor para las PyMEs sobre la importancia del proceso de la innovación, que está constituido de ciertos factores clave para el desarrollo de nuevos productos. Dicha investigación ofrece un aporte a las ciencias sociales administrativas, esperando que deje una trascendencia en el extenso campo del conocimiento.

En este apartado se presenta de manera contextual y como marco de referencia la influencia de los modelos de innovación exógenos basados en el enfoque de la escuela estructuralista. Se enfoca en la influencia de los modelos de proceso de innovación para el desarrollo de nuevos productos, su evolución en el tiempo y su impacto en la economía de las empresas dedicadas a la manufactura de productos.

\section{Metodología}

La industria manufacturera a partir de los años 1940's hasta la actualidad ha sufrido muchos cambios radicales desde la perspectiva de cómo se concebían los negocios en aquella época (Rothwell, R. 1994). La exigencia de los consumidores es cada vez más compleja de cómo el cliente y consumidor eran a partir de los 1940's. Han transcurrido crisis económicas a lo largo de la historia y algunos de éstas han ocasionado cambios en la forma de manufacturar, comercializar y desarrollar la introducción de productos al mercado. El mercado, por su parte, se ha hecho cada vez más complejo, ya que las necesidades de los consumidores cambian radicalmente cada vez más rápido por el incremento de competencia entre las empresas que conforman la industria. La innovación en el producto ahora es clave, y se ha hecho notar ya que el retener a los clientes es cada vez más difícil por la inmensa gama de productos que se ofrecen en el mercado, y por la inmensa cantidad de empresas que ofrecen los mismos productos.

Es por eso, que en la actualidad se escucha el tema de Innovación en todas partes, en las escuelas, en empresas, en los medios de comunicación, en anuncios publicitarios acerca de algún determinado producto, y hasta en los gobiernos de todos los países tratan de impulsar la innovación a través de 
productos y/o servicios como parte medular para el éxito en el crecimiento económico de las empresas, y éste a su vez, del país. La innovación es un tema actual y ha dejado de ser una estrategia puntual de la empresa para convertirse en un proceso clave, orgánico y sistemático de la misma organización (Arraut, 2007).

1.- Los modelos lineales cuya influencia se extiende años posteriores a la segunda guerra mundial (años 1940's) hasta mediados de 1960's, se caracterizan por su concepción lineal en el proceso de innovación de producto. La industria empieza a crecer y expandirse rápidamente en la mayoría de los mercados. Las compañías producen conforme al tipo de tecnología de maquinaria que existía en esa época y los consumidores se tenían que conformar con los productos que llegaban al mercado. Estas generaciones de modelos por lo tanto inician su proceso con el empuje de la tecnología hasta su etapa de venta del producto.

2.- Los modelos por etapas o secuenciales se desarrollan a partir de finales de los 1960's hasta mediados de los 1970's. En ese tiempo la industria manufacturera seguía creciendo fuertemente en muchos países y las compañías se esmeraban en la búsqueda de crecer pero en forma orgánica, y empieza a existir mucha competencia en el mercado. En este momento es cuando las compañías centran sus estrategias en focalizarse en las necesidades del mercado y ganar, de esta manera, una mayor participación del mismo. En esta generación de modelos empiezan a incluir las necesidades del mercado al inicio de su proceso para posteriormente desarrollar productos, y terminar en su etapa de comercialización.

3.- Los modelos Interactivos o Mixtos se desarrollan a partir de finales de la década de los 1970's hasta mediados de los 1980's. La necesidad de entender la lógica del proceso de innovación y las bases de las innovaciones exitosas era necesaria para conseguir reducir la incidencia de fallos y el despilfarro de recursos, ya que en esa época aparecieron niveles altos de inflación y las estrategias apuntaban en reducción de costos y control de estos a través de la organización. Fue en esta época cuando muchos autores trabajaron con estudios empíricos acerca del proceso de innovación. 
El modelo propuesto por Kline de cadena-eslabón fue un ejemplo de ello ya que no contemplaban la interacción que deberían de tener los departamentos que se encargaban de desarrollar un nuevo producto. Más aún, se incluyó en esta generación de modelos las retroalimentaciones que se deberían de tener a lo largo del proceso de desarrollo del producto con todas las personas relacionadas en la introducción del mismo al mercado, terminando en la estrategia de comercialización del mismo.

4.- Los modelos Integradores se desarrollan a partir de mediados de los años 1980's hasta mediados de los 1990's, y son conocidos por tratar de explicar la situación por la que se estaba pasando en ese tiempo con la rápida recuperación de la economía. Las empresas empiezan a enfocarse solamente en sus "core business", ya no le apuestan tanto a la diversificación. Se le apuesta al crecimiento a través de estrategias de tecnología (tecnologías de información; TI) basadas en la manufactura. Empiezan las alianzas entre compañías y no solo grandes empresas, si no también pequeñas y medianas empresas.

Los ciclos de vida de los productos son cada vez menores, lo que requería de mayor velocidad en la introducción de nuevos productos. Las compañías japonesas introducen estrategias en su manufactura como justo a tiempo (JIT), incluyen a los proveedores en el proceso de desarrollo de. Por esta razón se le denominó a esta generación como la de "enfoque rugby" en el cual el desarrollo de producto contrasta con el enfoque tradicional de carácter secuencial y representa la idea de que un grupo trabaje, pasando las decisiones y avances del proyecto hacia atrás y hacia delante, en la etapa de concepción del producto.

Bajo este enfoque, el proceso de desarrollo de producto tiene lugar en un grupo multidisciplinar cuyos miembros trabajan juntos desde el comienzo hasta el final de cada proyecto. Se le apuesta mucho al diseño de producto para eficientizar la manufactura. Muchas empresas, siguen apostando mucho a estos modelos de innovación por la alta productividad que se obtienen por el enfoque que le dieron a esta 4ta generación de modelos.

5.- El modelo en Red aparece a partir de finales de los 1990's y principios de los 2000's, de hecho es el que sigue vigente. Este tipo de modelos de innovación tiene lugar dentro y fuera de las empresas sugiriendo que la 
innovación es un proceso distribuido en red, las tendencias estratégicas están comprometidas con la acumulación tecnológica (estrategia tecnológica); las empresas continúan estableciendo redes estratégicas; la velocidad por llegar al mercado sigue siendo un factor de competitividad clave (estrategia por tiempo de respuesta); persisten los esfuerzos por lograr una mejor integración entre las estrategias de producto y las de producción (diseño para la manufactura de producto).

Las empresas muestran cada vez una mayor flexibilidad y adaptabilidad (organizacional, productiva y en productos); donde las estrategias de producto se enfatizan en la calidad y el rendimiento. Además se caracterizan por la utilización de sofisticadas herramientas electrónicas (TI) que permiten a las empresas incrementar la velocidad y la eficiencia en el desarrollo de nuevos productos, tanto internamente (distintas actividades funcionales), como externamente entre la red de proveedores, clientes y colaboradores al desarrollo del proyecto. Así mismo se le apuesta mucho en herramientas para el procesamiento de datos electrónicos para la mejor toma de decisiones.

Además de lo anterior, se decidió estudiar a la innovación pero enfocada al sector de las PyMEs por el grado de importancia en la economía mundial, como por citar un ejemplo:

- $\quad$ En USA las PyMEs en 2007, representaron el 99\% de las empresas registradas, empleando al $75 \%$ de la fuerza laboral y generando el 40.9\% del PIB. (http://clasica.xornal.com/article.php?sid=20070108011451)

- $\quad$ En la Unión Europea en 2006 (conformada por 27 países), las PyMEs representan el $99.8 \%$ de las empresas registradas, empleó al $67.4 \%$ de la fuerza laboral y generó el $57.7 \%$ del PIB total. (http://epp.eurostat.ec.europa.eu/portal/page/portal/eurostat/home/).

- En México las PyMEs son un sector que representa el $99.7 \%$ de empresas registradas, las cuales generan el $52 \%$ del PIB nacional, además contribuyen con el $71.9 \%$ de los empleos (OECD, 2005).

Y por lo tanto, conforme a los datos anteriores se ve la importancia de este sector por lo que la investigación busca estudiarla ya que es considerada para el aparato productivo o económico de cualquier país en el mundo. 
Un dato interesante de las PyMEs en México, es el señalar que en el reporte "La PyME y los Empresarios" de Nacional Financiera, se menciona los índices de mortandad por años trabajados de este tipo de empresas y además, las principales causas de muerte de las mismas.

Los índices de mortandad de las PyMEs se describen como sigue (Nacional Financiera, 2004):

- El 10\% solamente de las empresas que cumplen 10 años en el mercado, logran tener crecimiento, madurar y lograr éxito en el mercado.

- $\quad$ El $75 \%$ de las nuevas empresas deben de cerrar sus operaciones con tan sólo dos años de actividad económica.

- $\quad$ El 50\% de las empresas quiebran con tan solo un año de actividad.

- $\quad$ El $90 \%$ de las empresas mueren antes de cumplir los 5 años de vida.

En el mismo reporte, se muestran que los factores de causa de muerte de las PyMEs en México son derivados por:

- Problemas Administrativos y Fiscales.

- $\quad$ Incapacidad de cumplir los requisitos que obliga Hacienda.

- $\quad$ Deficiencias en la Comercialización y Errores en la compra de Insumos

- $\quad$ Falta de productividad en las áreas de Producción (Manufactura).

- Falta de re-Financiamiento.

- Falta de Nuevos Productos diferenciados o Innovadores.

Conforme a lo antes descrito, sobre las cinco generaciones de modelos de gestión de la innovación y la situación de las PyMEs en México, la cual tienen como una de sus causas de muerte la falta de nuevos productos Innovadores en el mercado, se observa que hay un campo de la investigación que no ha sido estudiado o explorado hasta el momento para el sector en México. Por lo tanto la pregunta de investigación:

¿Cuales son los factores clave que permiten integrar un proceso de gestión de la innovación más efectivo en el desarrollo de nuevos productos para las PyMEs?

Este propósito se traducirá a través de los siguientes objetivos que a continuación se describen. 


\section{Objetivos de la investigación}

Objetivo general:

Analizar los factores clave que permiten mejorar el proceso de gestión de la innovación para el desarrollo de nuevos productos en las PyMEs para que sean más competitivas, e Integrar estos factores en un modelo. (caso de estudio sector productivo de tecnologías de información en Nuevo León).

Objetivos específicos:

Analizar y revisar la literatura para encontrar teorías y autores que hayan estudiado sobre el proceso de gestión de la innovación de producto.

Identificar y seleccionar el conjunto de factores involucrados en el proceso de la Innovación en base a los modelos teóricos existentes.

Integrar en un modelo, la selección de los factores clave de los modelos teóricos existentes que resulte del análisis de literatura.

Elaborar y aplicar la encuesta a la muestra seleccionada a fin de validar los factores clave dentro del modelo y su importancia relativa individualmente de cada uno de estos factores.

Analizar los resultados, establecer conclusiones y recomendaciones que permitan validar el modelo propuesto.

\section{$\underline{\text { Hipótesis general y específicas de la investigación }}$}

Derivado de lo que se analizó en la problemática y en los objetivos, el trabajo en curso se encamina a definir las hipótesis de estudio:

$\mathrm{Ha}=$ "Los factores clave necesarios para integrar un proceso de gestión de la innovación que permita mejorar el desarrollo de nuevos productos para PyMEs son: Orientación al Mercado (OM), Creatividad Organizacional (CR), Investigación \& Desarrollo (lyD), Diseño de Producto (DP), Eficiencia Operacional (EO) y Eficiencia Comercial (EC)". 
Las variables independientes impactan positivamente en el proceso de gestión de la innovación en el desarrollo de nuevos productos para PyMEs, siendo éstas la:
$\mathrm{H}_{1}$ : La Orientación al Mercado (OM)
$\mathrm{H}_{2}$ : La Creatividad (CR)
$\mathrm{H}_{3}$ : La Investigación \& Desarrollo (lyD)
$\mathrm{H}_{4}$ : El Diseño de Producto (DP)
$\mathrm{H}_{5}$ : La Eficiencia Operacional (EO)
$\mathrm{H}_{6}$ : La Eficiencia Comercial (EC)

\section{Acerca de la industria de tecnologías de la información}

Habiendo cubierto la parte contextual de la investigación, es tiempo de entrar a explorar la industria sobre la cual éste artículo aplicará su instrumento de medición, Tecnologías de la Información.

Para entrar en contexto, se dice que en el ámbito de las Ciencias Sociales se dice que han existido 3 revoluciones industriales: la primera comienza en el siglo XVIII, la segunda se da a principios del siglo XX y la tercera viene a significar el cambio actual. De manera general en cada una de estas revoluciones se ha pasado de procesos agrícolas a industriales, de tecnologías escasas a tecnologías necesarias, de gobiernos autocráticos a gobiernos democráticos y de bienes y productos materiales a inmateriales (Lucas, 1999).

La Secretaría de Economía y el Gobierno Federal han impulsado iniciativas para promover la productividad y fomentar que la economía mexicana escale hacia sectores de alto valor agregado. Es por ello que una de las principales estrategias para fomentar la innovación y la competitividad, así como el posicionamiento internacional es apoyar el sector de Tecnologías de la información. La Secretaría de Economía (2009) comenta que el Plan Nacional de Desarrollo 2001 - 2006 plantea el fomento a la industria y el mercado de Tecnologías de la Información (TI) como estrategia para aumentar la competitividad del país. Las TI tienen un efecto transversal en toda la economía, razón por la cual impactan positivamente la competitividad de todos los sectores. Dado el gran potencial con que cuenta México para desarrollar esta industria, la Secretaría, en coordinación con empresas del 
sector, se diseña el Programa para el Desarrollo de la Industria del Software en México llamado; PROSOFT.

Según la Secretaría de Economía asevera que el país (http://www.economia.gob.mx/?P=1128):

1. México tiene un nivel de gasto en tecnologías de la información y comunicaciones (TIC) de $3.2 \%$ del PIB, ubicándose en el lugar 50 a nivel mundial.

2. Este rezago es aún mayor en términos de gasto en software, que es 6 veces inferior al promedio mundial y 9 veces menor que el de EUA.

3. Países como la India, Irlanda y Singapur han sido exitosos en desarrollar su industria de software como motor de su crecimiento económico.

4. México cuenta con un gran potencial para desarrollar esta industria dada su cercanía geográfica y el mismo uso de horario con el mercado de software más grande del mundo (EUA); la red de tratados comerciales más extensa de mundo; y afinidad con la cultura de negocios occidental.

Según para la Secretaria de Economía los beneficios de invertir en el sector de TI son:

1. Las $\mathrm{TI}$ son capaces de elevar el PIB de cualquier país hasta en $5 \%$.

2. La correlación entre la adopción de TICs y Competitividad de países es $92 \%$.

3. El uso de $\mathrm{TI}$ pueden incrementar hasta un $20 \%$ la productividad en las organizaciones.

4. Los sueldos en el sector de TI son de 3 a 5 veces mayores a los sueldos de manufactura.

La industria del software interviene en todos los procesos que habilitan a la "nueva economía", se le considera una industria blanca que no contamina y que genera fuentes de trabajo bien remuneradas. Entre los casos de éxito que México debe tomar en cuenta, están el de la India, donde se manufactura software, 0 el de Brasil donde el gobierno incentiva la creación de empresas con el fin de competir eficazmente con Estados Unidos, Irlanda y Canadá (Peñaloza, 2002). 
Aunque el enfoque de la industria de software en México se ha dirigido principalmente hacia el mercado interno, actualmente se mueve hacia los mercados internacionales. Se podría ser uno de los protagonistas de la nueva economía digital, mediante acciones coordinadas, si se consolida el concepto de fábricas de software para convertirse en exportador de este servicio. El potencial de ingresos, a nivel país, sería superior a los cinco mil millones de dólares anuales en el mediano plazo y los desarrolladores mexicanos podríamos construir una marca con reconocimiento global, además que el $35 \%$ de este monto se exporta (A.T. Kearney, 2009).

Según la CANIETI en su informe de febrero 2010, en colaboración con la Secretaría de Economía, SELECT e IDC, la industria de TI en México a sumado los siguientes valores a nivel país:

1. Valor de mercado durante 2009: $\$ 14,507.0$ Musd.

2. Crecimiento anual de $6 \%$, dos dígitos en los últimos 5 años.

3. Auge en el modelo BPO's (denominado Tercerización de Procesos de Negocios)

4. Comparativa del mercado de Tl en el Mundo y en México.

Mercado de TI World Wide: $20092010 \quad$ Crecimiento

IT Services total

$\$ 583,167.0$

$\$ 599,058.7 \quad 2.73 \%$

Packaged software total

$\$ 304,791.7$

$\$ 316,538.8$

$3.85 \%$

Hardware total

$\$ 559,466.5$

$\$ 574,728.0$

$2.73 \%$

Total

$\$ 1,447,425.3$

$\$ 1,490,325.4$

$2.96 \%$

Mercado de TI en México:

IT Services

$\$ 3,388.5$

$\$ 3,646.9$

$7.63 \%$

Packaged software

$\$ 1,888.1$

$\$ 1,955.7$

$3.58 \%$

Hardware

$\$ 9,230.6$

$\$ 9,663.4$

$4.69 \%$

Total

$\$ 14,507.2$

$\$ 15,266.0$

$5.23 \%$

Cifras en millones de dólares, Fuente: IDC Black Book Q4 2009. 
Se estima que en Nuevo León alrededor de 350 empresas conforman la industria del software y cerca del 20 por ciento se encuentran formalmente estructuradas, ya sea como subsidiarias de grandes empresas internacionales, como organizaciones netamente mexicanas, 0 una combinación de ambas (csoftmty, 2009). Un aspecto importante relacionado con este hecho es que más del 90 por ciento del universo de este sector lo integran microempresas, la mayoría de las cuales carece de estructura y de un enfoque formal en sus actividades de venta y elaboración de contratos, además de que prestan servicios en forma aislada y en actividades muy específicas a diversos sectores.

Algunas cifras básicas de la industria del software en el estado de Nuevo León (csoftmty, 2009):

1. 350 empresas ( $9 \%$ grandes, $17 \%$ medianas, $31 \%$ pequeñas, $43 \%$ microempresas), y que generaron 7,000 empleos.

2. Facturación en 2008 del cluster de $\$ 320$ Musd y promedio por empleado de $\$ 381,000$ usd, así como sueldo promedio de $\$ 25,885.00$ pesos por mes.

Se reconoce a esta industria como "limpia" y generadora de empleos, basada en tecnología e innovación con alto valor agregado y alta remuneración comparada con otras industrias. Además se cuenta, dentro del cluster, con una participación de gente tanto de nivel técnico, como profesionistas y postgrados a nivel maestría y doctorado. Son organizaciones de baja inversión, ya que el capital humano es su activo principal, por lo que el tema de educación es fundamental. Después de haber visto algunos datos que respaldan la importancia de la industria de TI en México y en Nuevo León, es por esta razón que se estudiará esta Industria.

Resumiendo la primera parte del capítulo, se puede decir que se presentaron de manera detallada la influencia de los modelos de gestión de la innovación para el DNP, su evolución en el tiempo e impacto en la economía de las empresas dedicadas a la manufacturación de productos. A su vez, se profundizará más adelante en este estudio el tema de la creatividad y sus modelos de gestión a través del tiempo y, terminando con las matrices comparativas de las variables que componen los modelos, tanto de Innovación como de Creatividad. 
Por lo tanto, después de haber conocido las cinco grandes generaciones de modelos de innovación de producto, se puede deducir que el mercado y las necesidades de los consumidores son cada vez más complejas y que debemos estar preparados como empresa para irnos adaptando en estos cambios.

Para algunas compañías los modelos lineales son la solución a sus problemas de innovación, mientras que otras organizaciones los modelos integradores, o de sistema en red les pudiera resultar con más beneficio, o el modelo que se propone en este trabajo puedira abarcar todas las necesidades que necesite la PyME para ser más innovadores en su propuesta de valor en el mercado. Lo importante, es que se debe de estar consciente de la existencia de éstos modelos y poderlos implementar en la organización dentro de las limitaciones que pueda tener cada empresa sin importar su tamaño.

Se visualiza en forma de mapa del tiempo las generaciones de los modelos.

\section{Figura 1. Mapa de la línea del tiempo para los MGI de producto.}

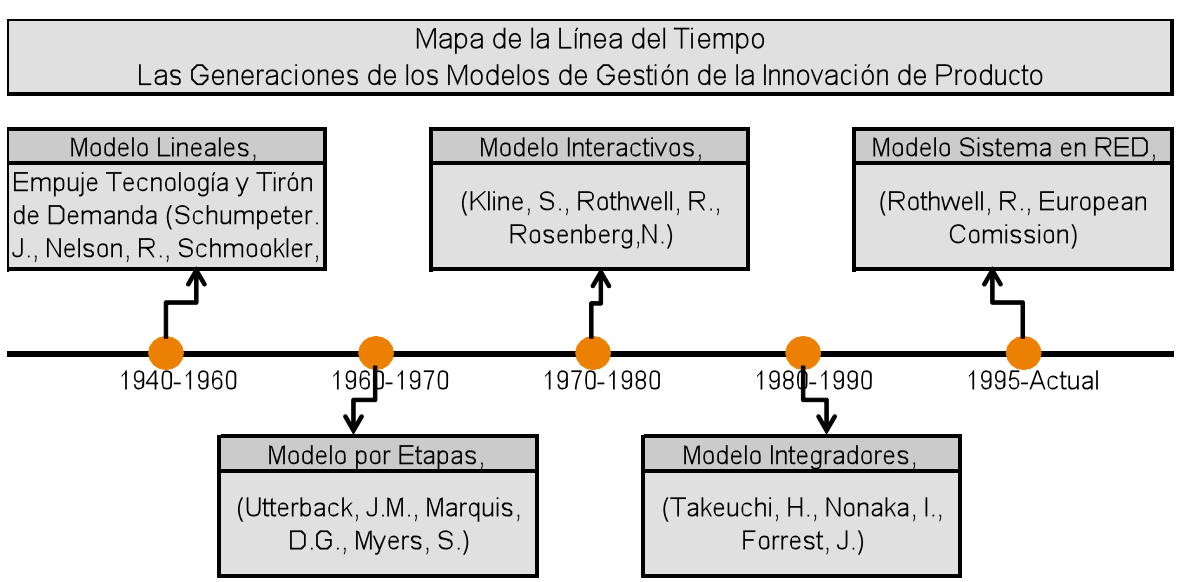

Fuente: Elaboración Propia a partir de la época en que han aparecido los modelos 
Tabla 2. Matriz comparativa de las variables que conforman los modelos de gestión de la innovación, incluyendo la propuesta de investigación.

\begin{tabular}{|c|c|c|c|c|c|c|}
\hline Grandes Rubros & \begin{tabular}{|l|} 
1ra. Generación- \\
Modelos Lineales
\end{tabular} & $\begin{array}{l}\text { 2da. Generación- } \\
\text { Modelos por } \\
\text { Etapas o Deptos. }\end{array}$ & $\begin{array}{l}\text { 3ra. Generación- } \\
\text { Modelos Mix tos o } \\
\text { Interactivos }\end{array}$ & $\begin{array}{l}\text { 4ta. Generación- } \\
\text { Modelos } \\
\text { Integradores }\end{array}$ & $\begin{array}{l}\text { 5ta. Generación- } \\
\text { Modelos en RED }\end{array}$ & $\begin{array}{c}\text { Modelo de } \\
\text { Innovación para } \\
\text { PyMEs }\end{array}$ \\
\hline $\begin{array}{l}\text { Orientación al Mercado (OM) } \\
\text { (Clientes) }\end{array}$ & & $x$ & $x$ & $x$ & $x$ & $x$ \\
\hline $\begin{array}{l}\text { Creatividad Organizacional } \\
\text { (CR) }\end{array}$ & & & & & & $x$ \\
\hline $\begin{array}{c}\text { Investigación \& Desarrollo } \\
(\mathrm{lyD})\end{array}$ & $x$ & $x$ & $x$ & $x$ & $x$ & $x$ \\
\hline Diseño de Producto (DP) & $x$ & $x$ & $x$ & $x$ & $x$ & $x$ \\
\hline $\begin{array}{c}\text { Eficiencia Operacional (EO), } \\
\text { (Operaciones, Finanzas) }\end{array}$ & $x$ & $x$ & $x$ & $x$ & $x$ & $x$ \\
\hline Eficiencia Comercial (EC) & $x$ & $x$ & $x$ & $x$ & $x$ & $x$ \\
\hline $\begin{array}{l}\text { Alianzas Estratégicas, } \\
\text { Proveedores }\end{array}$ & & & & $x$ & $x$ & \\
\hline $\begin{array}{c}\text { Distribuidores, } \\
\text { Universidades, Gobierno }\end{array}$ & & & & & $x$ & \\
\hline $\begin{array}{l}\text { Conocimiento Público, } \\
\text { Competidores, Sociedad }\end{array}$ & & & & & $x$ & \\
\hline
\end{tabular}

Fuente: Elaboración Propia a partir de los datos anteriores.

Se muestra en la tabla 2, a forma de resumen, las cinco generaciones de modelos de proceso de la innovación de producto. Se plasma el modelo propuesto de proceso de innovación para PyMEs al final de dicha tabla. Se coloca una "X" para visualizar cual es el proceso que se está tomando en cuenta en cada modelo. Se puede observar que para la propuesta de modelo de PyMEs hay un constructo que no se ha tomado en cuenta en ninguna de las generaciones pasadas. El elemento de Creatividad Organizacional con el enfoque de generación de ideas en forma orgánica y sistemática tanto al interior como al exterior de la organización es la propuesta de valor en la cual se sustenta este proyecto de investigación.

Es tiempo de profundizar más en esta variable y justificar el porqué debe ser considerada en este nuevo modelo de gestión de innovación para las PyMEs. La variable de creatividad organizacional debe ser considerada y vista como un proceso operacional más de la compañía, como lo es el proceso de compras o cobranza de cualquier organización (Bono, E. 1994).

En la actualidad, el término creatividad ha cobrado un papel relevante en las materias como educación, economía y política. Desde el enfoque en el ámbito económico o empresarial, se demandan personas con un determinado perfil psicológico, en el que, además de una serie de 
conocimientos técnicos, calidad humana, motivación, perseverancia, y toma de decisiones, la capacidad creativa o innovativa ocupa un lugar importante (Peralta, F. 1998).

La creatividad de los individuos es el punto de partida para la innovación, es la semilla para crear innovación (Amabile y otros 1996). La relación entre creatividad e innovación lleva a gestionar adecuadamente a las empresas que desean ser innovadoras. Muchos autores señalan que la creatividad es la materia prima de la innovación visto desde la perspectiva de los negocios y que ésta se debe, en cierta manera, de gestionar para obtener resultados positivos en la resolución de problemas.

Otra destacada pensadora sobre esta materia es Amabile que en 1988 , encontró en un estudio que realizó con 165 científicos que existen algunos factores del entorno que facilitaban la creatividad mientras que otros factores la inhibían.

El resultado del estudio arrojó los siguientes datos sobre los factores que facilitan la creatividad:

1. Libertad y Control del trabajo.

2. Una buena dirección que establece metas, evita distracciones y no es demasiado estricta.

3. Recursos suficientes.

4. Estimulación de nuevas ideas en el entorno laboral.

5. Colaboración entre las divisiones del trabajo.

6. Reconocimiento del trabajo creativo.

7. Disponer de suficiente tiempo para pensar.

8. La existencia de problemas desafiantes.

9. Sentido de la urgencia de que el trabajo sea realizado.

A su vez, los factores que limitan la creatividad, en este estudio, fueron definidos como:

1. Pobres recompensas y pobre comunicación

2. Formalidades burocráticas y falta de libertad de accionar.

3. Evaluación poco realista e inadecuada (Apatía).

4. Insuficiencia de recursos y presión debida a la falta de tiempo. 
5. Falta de voluntad para asumir el riesgo que supone el cambio.

6. El carácter defensivo de la organización y la competitividad.

A continuación se muestra, en forma de resumen, una matriz comparativa de los modelos de creatividad que van desde los años 1920's hasta los 1990's.

\section{Tabla 3. Matriz comparativa de los modelos de creatividad en el tiempo}

\begin{tabular}{|c|c|c|c|c|c|c|c|c|c|}
\hline Grandes Rubros & \begin{tabular}{|c|}
$\begin{array}{c}\text { Modelo de } \\
\text { "Wallas 1926" }\end{array}$ \\
\end{tabular} & 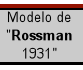 & $\begin{array}{c}\text { Modelo de } \\
\text { "Osborn 1953" } \\
\text { (brainstorming) } \\
\end{array}$ & \begin{tabular}{|c|} 
Modelo de \\
"Koberg y \\
Bagnall 1981" \\
\end{tabular} & $\begin{array}{c}\begin{array}{c}\text { Modelo de } \\
\text { "Amabile } \\
\text { 1983" }\end{array} \\
\end{array}$ & $\begin{array}{c}\text { Modelo de } \\
\text { "Bandrowski } \\
1985 " \\
\end{array}$ & $\begin{array}{c}\text { Modelo de } \\
\text { "Barron 1988" } \\
\end{array}$ & $\begin{array}{l}\text { Modelo de } \\
\text { "Fritz 1991" } \\
\end{array}$ & $\begin{array}{l}\text { Modelo de "Parnes } \\
\text { 1992, Isaksen y } \\
\text { Trefflinger 1985" }\end{array}$ \\
\hline \begin{tabular}{|c|} 
Orientación / Comprobación \\
de la ldea
\end{tabular} & & & $\mathrm{x}$ & $\mathrm{X}$ & $\mathrm{x}$ & $\mathrm{x}$ & & $x$ & $x$ \\
\hline $\begin{array}{c}\text { Preparación / Concepción de } \\
\text { la ldea }\end{array}$ & $x$ & $x$ & $x$ & & $x$ & $x$ & $x$ & $x$ & \\
\hline Análisis de Necesidad & & & $x$ & $x$ & & $x$ & & $x$ & $x$ \\
\hline $\begin{array}{c}\text { Incubación / Gestación / } \\
\text { Estudio de Soluciones }\end{array}$ & $\mathrm{X}$ & $\mathrm{X}$ & $\mathrm{X}$ & $x$ & $x$ & & $x$ & $\mathrm{x}$ & $\mathrm{x}$ \\
\hline Iluminación / Parto & $x$ & $x$ & & $x$ & $x$ & $x$ & $x$ & & $x$ \\
\hline $\begin{array}{c}\text { Formulación / Idealización } \\
\text { de Soluciones }\end{array}$ & & $\mathrm{X}$ & $\mathrm{X}$ & $\mathrm{X}$ & $\mathrm{X}$ & & & $\mathrm{x}$ & \\
\hline Construcción del Concepto & & & & & & $x$ & & $x$ & \\
\hline $\begin{array}{l}\text { Análisis Critico de las } \\
\text { Soluciones / Sintesis }\end{array}$ & & $\mathrm{x}$ & $x$ & & $\mathrm{x}$ & $\mathrm{x}$ & & & $x$ \\
\hline $\begin{array}{c}\text { Planeación e Implementar } \\
\text { Solución }\end{array}$ & & & & $x$ & & $x$ & & $\mathrm{x}$ & \\
\hline $\begin{array}{l}\text { Verificación / Crianza / } \\
\text { Aceptación de la Solución }\end{array}$ & $\mathrm{X}$ & $\mathrm{X}$ & $\mathrm{X}$ & $x$ & & $x$ & $x$ & $\mathrm{x}$ & $x$ \\
\hline
\end{tabular}

Fuente: Elaboración Propia a partir de datos anteriores.

En la tabla 3, se plasma el resultado del análisis de las diferentes propuestas de modelos propuestos de gestión de la creatividad, en el cual se expresa la relación de las variables que conforman dichos modelos. Se coloca una " $X$ " para visualizar el proceso que cubre dicho modelo.

A continuación se conjuntan las variables que son propuestas en la hipótesis de esta investigación para dar nacimiento a un nuevo modelo, siendo éste la propuesta de valor del estudio.

En la figura 2, se muestra el diagrama anterior se puede observar cómo se colocan las seis variables independientes del lado izquierdo (orientación al mercado, creatividad organizacional, investigación \& desarrollo, diseño de producto, eficiencia operacional, eficiencia comercial y que éstas afectan todas ellas de manera positiva a la variable dependiente 
(lado derecho) que es la gestión de la innovación en el desarrollo de productos para las PyMEs.

Figura 2. Factores clave en forma de modelo.

Variables

Independientes

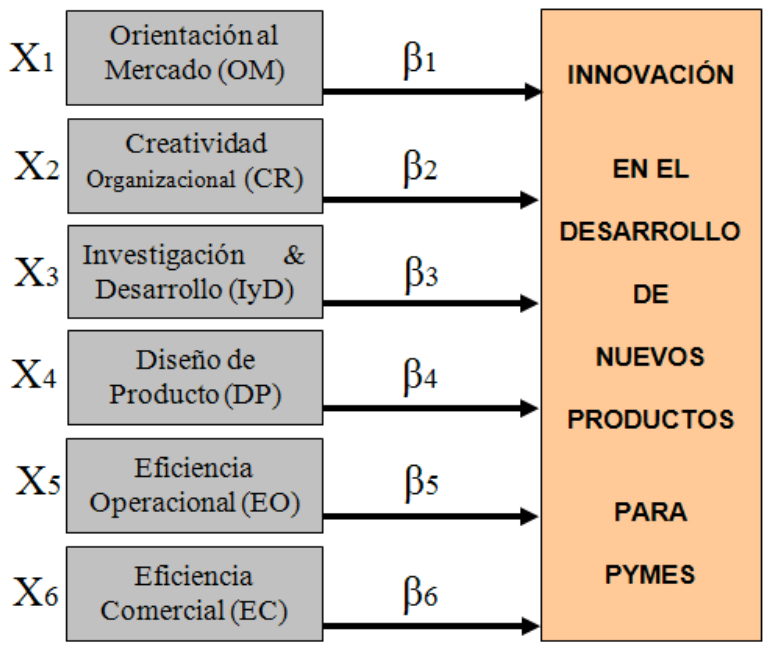

Fuente. Elaboración Propia.
Variable

Dependiente

NOVACIÓN

EN EL

DESARROLLO

DE

NUEVOS

PRODUCTOS

PARA

PYMES

\section{Diseño y tipo de la investigación}

Dada la naturaleza del trabajo de investigación propuesto, el diseño en el proceso de la investigación será NO experimental ya que no se hace variar intencionalmente las variables independientes, por lo que aquí se observan solamente los hechos como son. Se considera a su vez de tipo Explicativo ya que el resultado de la correlación de las variables generarán un entendimiento hacia el fenómeno analizado, por lo cual se podrá explicar las causas del impacto de éstos en la variable dependiente.

El tipo de muestra que se utilizará en este trabajo de investigación será "probabilística" ya que por medio del método random (en una hoja de cálculo) se escogerán las 53 empresas de las 119 de la población meta serán encuestadas para dicho estudio. La investigación refiere que es un estudio "NO Paramétrico", ya que se mandará la información a la población total del 
estudio ya que el porcentaje de obtener la información con respecto a los que nos pide la Nóptima nos da un margen muy pequeño para poder solamente enfocarnos en algunas cuantas.

Una vez vaciada la información en una hoja de cálculo se procederá a realizar sus análisis estadísticos en el SPSS correspondientes como sigue:

- Análisis de estadística Descriptiva e Inferencial.

- Dentro de la segunda, se procede a realizar un análisis de fiabilidad; alfa de cronbach (para analizar el impacto del ítem en cada variable).

- Ponderación de las preguntas en la encuesta y de cada variable.

- Análisis de regresión lineal múltiple.

- Análisis de las ANOVAS.

- Obtener algunos medios gráficos (multicolinealidad).

- Y por último la Interpretación de los resultados estadísticos de la investigación, tanto cualitativos como cuantitativos.

Utilizando para este efecto el Software SPSS versión 15.

Ecuación general de investigación:

$$
Y=a+X 1 \beta 1+X 2 \beta 2+X 3 \beta 3+X 4 \beta 4+X 5 \beta 5+X 6 \beta 6+e
$$

\section{Resultados}

En este capítulo se presentan los resultados de la investigación del total de las empresas encuestadas utilizando la estadística inferencial.

Los resultados del análisis de fiabilidad (alfa de cronbach), en la siguiente tabla se puede observar los resultados con las 53 empresas, comparando la relación de las preguntas por cada constructo y en forma conjunta.

En la tabla 4, se muestran los siguientes resultados estadísticos con relación a las variables de esta investigación: 
1. Un valor de alfa de 0.955 lo que muestra buena correlación entre las 53 preguntas que conforman la encuesta, por lo que no hay necesidad de eliminar ninguna, por lo tanto se deduce que la encuesta es aceptable.

2. En el análisis individual de alfa para cada una de las variables, las variables (Orientación al Mercado, Creatividad Organizacional, Investigación \& desarrollo, Eficiencia Operacional, Eficiencia Comercial) obtuvieron valores por arriba del $85 \%$, y la variable de Diseño de Producto con un valor de $68 \%$. Todos estos resultados son aceptables y favorables por lo que se deduce que las preguntas de cada variable no están correlacionadas entre sí, son totalmente independientes una de la otra (una no depende de la otra), por lo tanto de vuelve a corroborar que la encuesta es aceptable.

3. La significancia para las seis variables nos da como resultado abajo del $5 \%$, de hecho son casi cero, por lo que se considera aceptable.

4. Todas las pruebas de Friedman (prueba F) dieron arriba de 2.2 por lo que se considera muy dentro de los parámetros requeridos. Por lo que aquí podemos deducir que nuestra encuesta está listo para su análisis de regresión lineal.

Tabla 4. Resultados del análisis de fiabilidad de la muestra.

\begin{tabular}{|c|c|c|c|c|c|c|c|c|c|c|}
\hline Variables & $\mathrm{N}$ & $\begin{array}{c}\text { Items } \\
\text { Iniciales }\end{array}$ & $\begin{array}{c}\text { Media } \\
\text { Global }\end{array}$ & Media & Varianza & $\begin{array}{c}\text { Desviacio } \\
\text { n Típica }\end{array}$ & $\begin{array}{c}\text { Alfa } \\
\text { Cronbach }\end{array}$ & gl & $\begin{array}{c}\text { Prueba "F" } \\
\text { Friedman }\end{array}$ & $\begin{array}{c}\text { Signifi- } \\
\text { cancia }\end{array}$ \\
\hline OM & 53 & 9 & 3.807 & 34.260 & 47.083 & 6.861 & 0.859 & 8.000 & 5.772 & 0.000 \\
\hline CR & 53 & 9 & 3.245 & 29.207 & 51.668 & 7.188 & 0.883 & 8.000 & 22.796 & 0.000 \\
\hline I\&D & 53 & 9 & 3.232 & 29.094 & 76.087 & 8.722 & 0.901 & 8.000 & 9.542 & 0.000 \\
\hline DP & 53 & 7 & 3.070 & 21.490 & 22.255 & 4.717 & 0.684 & 6.000 & 22.144 & 0.000 \\
\hline EO & 53 & 10 & 3.179 & 31.792 & 62.398 & 7.899 & 0.863 & 9.000 & 2.232 & 0.019 \\
\hline EC & 53 & 9 & 3.519 & 31.672 & 53.914 & 7.342 & 0.885 & 8.000 & 10.190 & 0.000 \\
\hline TOTAL & 53 & 53 & 3.349 & 177.528 & 1133.79 & 33.671 & 0.955 & 52.000 & 11.789 & 0.000 \\
\hline
\end{tabular}

Fuente: Elaboración propia.

De la tabla 5 , se completa la sustitución de los valores de las betas en la ecuación general del estudio:

$Y=0-0.049 X 1+0.390 X 2+0.819 X 3+0.073 X 4+0.103 X 5+0.028 X 6$. 
Tabla 5. Resultados de la regresión lineal múltiple a través del origen

\begin{tabular}{|c|c|c|c|c|c|c|c|c|c|}
\hline $\begin{array}{c}\text { Modelo } \\
\text { Completo }\end{array}$ & $\mathrm{N}$ & Variables & $\mathrm{R}$ & $\mathrm{R}^{2}$ & $\mathrm{R}^{2}$ corregida & $\mathrm{gl}$ & $\begin{array}{c}\text { Durbin- } \\
\text { Watson }\end{array}$ & $\begin{array}{c}\text { "F" en la } \\
\text { Anova }\end{array}$ & $\begin{array}{c}\text { Significancia en } \\
\text { la Anova }\end{array}$ \\
\hline Regresión & 53 & 1 & 0.903 & 0.816 & 0.812 & 1 & 2.026 & 230.112 & 0.000 \\
\hline
\end{tabular}

\begin{tabular}{|c|c|c|c|c|c|c|c|}
\hline \multicolumn{10}{|c|}{ Variables Excluidas } \\
\hline \multirow{2}{*}{ Modelo } & Beta Dentro & $\mathrm{t}$ & Sig & $\begin{array}{c}\text { Correlación } \\
\text { Parcial }\end{array}$ & \multicolumn{3}{|c|}{ Estadísticos de Colinealidad } \\
\cline { 6 - 8 } & & & & Tolerancia & FIV & $\begin{array}{c}\text { Tolerancia } \\
\text { Mínima }\end{array}$ \\
\hline OM & -0.049 & -0.208 & 0.836 & -0.029 & 0.065 & 15.341 & 0.065 \\
\hline CR & 0.390 & 1.525 & 0.133 & 0.209 & 0.053 & 18.904 & 0.053 \\
\hline DP & 0.073 & 0.274 & 0.785 & 0.038 & 0.051 & 19.538 & 0.051 \\
\hline EO & 0.103 & 0.305 & 0.762 & 0.043 & 0.031 & 31.929 & 0.031 \\
\hline EC & 0.028 & 0.125 & 0.901 & 0.018 & 0.071 & 14.179 & 0.071 \\
\hline
\end{tabular}

Fuente: Elaboración Propia.

De la tabla 5, se muestran los siguientes resultados del análisis de las 53 empresas que se encuestaron de la industria de T.I. en Nuevo León:

1. Cabe señalar que los análisis sin considerar Beta 0 (partiendo del origen) consideran solamente las variables más significativas. Por lo que este estudio se obtuvo solamente aceptable la variable de investigación \& desarrollo. Las otras cinco variables fueron excluidas por el análisis en el SPSS. Estas variables se descartan por su baja significancia para el modelo.

2. Del análisis de coeficiente de determinación $\left(R^{2}\right)$ que establece el porcentaje de variación de la variable dependiente, resulta que una variable (investigación \& desarrollo) cumple con el porcentaje para que se considere aceptable con un valor de $81.6 \%$, como lo indica el valor de $\mathrm{R}^{2}$.

3. La correlación que existe de las seis variables independientes vs la variable dependiente es aceptable, como lo indica el Durbin-Watson con su valor de 2.026 (rango aceptable entre 1.5 a 2.5).

4. El análisis de varianza Anova compara las medias de cada variable para demostrar que existe independencia entre las variables independientes (que no estén correlacionadas; que una variable no dependa de la otra), por lo que se deduce que la variable investigación \& desarrollo muestra 
independencia vs las otras variables con su valor de 230.1. Además su significancia es prácticamente cero por lo que se considera aceptable.

5. El valor de la significancia de la tstudent es casi cero, por lo que también es suficiente para considerarse correcta. Esto significa que el resultado de cada variable es confiable y el error estimado es menor al $5 \%$, por lo que la variable (investigación \& desarrollo) es significativa. El valor de la $\mathbf{t}$ es 15.16 para la variable I\&D, por lo tanto es aceptable y es de gran relevancia ya que esta métrica evalúa si las variables difieren entre sí de manera significativa.

6. La multicolinealidad, que describe la correlación de las variables explicativas 0 independientes, es aceptable con su valor de FIV de 1, cuando el rango aceptable es de 1 a 10. Para este caso como fue solamente una variable toma el valor de 1.

7. La beta de la variable I\&D obtuvo un valor de 0.90 por lo que el impacto es muy alto de esta variable sobre la innovación de producto aparte de ser positivo (entre más investigación \& desarrollo, más innovación de producto).

8. Las variables que se excluyen del modelo fueron orientación al mercado, diseño de producto, eficiencia operacional, creatividad organizacional y eficiencia comercial. Estos obtuvieron valores muy bajos, por lo que no son significativos. Los impactos de estas variables sobre la dependiente (innovación de producto) no resultaron ser significativos por lo que el SPSS los excluyó por falta de peso para los análisis de regresión lineal múltiple.

Concluyendo este análisis de muestreo final, se validó que la variable investigación \& desarrollo es la significativa para el modelo propuesto. Se observó también que para la variable creatividad organizacional estuvo muy cerca de considerarse significativa, por lo que se puede deducir que los empresarios apenas la empiezan a valorar como elemento importante en este sector de la industria. Por lo tanto, en base a lo anterior se puede contestar las hipótesis del estudio.

Por lo que se observa en la figura 3, la única hipótesis aceptable es investigación \& desarrollo con un impacto positivo sobre la variable dependiente. El resto de las cinco hipótesis se rechazan para este estudio por su baja significancia. 
Figura 3. Matriz de resultados de las hipótesis de investigación.

\begin{tabular}{|l|c|c|c|c|}
\hline \multicolumn{1}{|c|}{ Hipótesis de Investigación } & Beta & $\begin{array}{c}\text { Signifi- } \\
\text { cancia }\end{array}$ & $\begin{array}{c}\text { Acepta o Rechaza } \\
\text { las Hipótesis }\end{array}$ & $\begin{array}{c}\text { Impacto sobre la } \\
\text { Innovación d/producto }\end{array}$ \\
\hline Orientación al Mercado & -0.049 & $\mathbf{0 . 8 3 6}$ & Rechaza & Impacto Negativo \\
\hline Creatividad Organizacional & 0.390 & $\mathbf{0 . 1 3 3}$ & Rechaza & Impacto Positivo \\
\hline Investigación y Desarrollo & 0.903 & $\mathbf{0 . 0 0 0}$ & Acepta & Impacto Positivo \\
\hline Diseño de Producto & 0.073 & $\mathbf{0 . 7 8 5}$ & Rechaza & Impacto Positivo \\
\hline Eficiencia Operacional & $\mathbf{0 . 1 0 3}$ & $\mathbf{0 . 7 6 2}$ & Rechaza & Impacto Positivo \\
\hline Eficiencia Comercial & $\mathbf{0 . 0 2 8}$ & $\mathbf{0 . 9 0 1}$ & Rechaza & Impacto Positivo \\
\hline
\end{tabular}

Fuente: Elaboración Propia.

\section{Conclusiones}

El presente estudio de investigación trata de aportar una herramienta más a las PyMEs del sector de T.I. en Nuevo León para mejorar la innovación de sus productos, a través del modelo de gestión de innovación propuesto.

La innovación, en las generaciones de los modelos de proceso de la innovación, resulta ser un proceso complejo porque cada uno de los modelos propuestos de generación en generación trata de mejorar a sus predecesores, realizando nuevas aportaciones a los mismos de forma que se ha ido perfeccionando el conocimiento sobre la forma en la que se implementa la innovación en la empresa que busca cumplir con las necesidades cambiantes de los consumidores tomando en cuenta la transformación que ha venido sufriendo los sectores económicos.

En la perspectiva teórica de la escuela de pensamiento reconstruccionista para la innovación (Kim, 2005), que se apoya en la teoría del crecimiento económico endógeno, donde la innovación en sus diferentes tipos emerge del interior de la organización, se obtuvo un avance en diferentes aspectos, ya que:

- Se planteó un modelo para explicar la importancia de la Innovación en el desarrollo de nuevos productos en el sector de las PyMEs por medio de seis variables las cuales son: el Orientación al Mercado, la Creatividad 
Organizacional, la Investigación \& desarrollo, el Diseño de Producto, la Eficiencia Operacional y por último la Eficiencia Comercial.

- Se conjuntó las variables anteriormente mencionadas en un proyecto que en base a la información analizada ningún estudio teórico en México se había hecho.

- Se propuso la variable Creatividad Organizacional como el constructo adicional, ya que los modelos de gestión teóricos existentes no la habían tomado en cuenta, y que afecta directamente y de forma positiva al proceso de innovación de producto. Esta variable busca la exploración y generación de ideas, a partir de ciertas necesidades del mercado, pero al interior de la organización a través de su gente. En esta etapa como la llama Forrest (1991) pre-innovación o de exploración, se generan ideas y se evalúan opciones, por lo que la creatividad resulta vital en la organización. Además de evitar el desperdicio que suele tener las organizaciones al no explotar el potencial creativo de la gente que forma parte de la organización.

La mayoría de los modelos estudiados parecen estar orientados hacia empresas de gran tamaño, que disponen de departamentos internos de lyD, dejando a un lado las empresas de menor tamaño que operan a través de procesos más informales y que no cuentan con departamentos propios de mercadotecnia, innovación, ingeniería o de investigación \& desarrollo. La ventaja que tienen las PyMEs sobre la grande empresa es que es más rápida a los cambios culturales de trabajo al contar con mayor flexibilidad para adaptarse a las nuevas tecnologías.

\section{Conclusiones en base a las hipótesis de investigación.}

Hipótesis \# 1: "La Orientación al Mercado impacta positivamente en el proceso de gestión de la innovación en el desarrollo de nuevos productos para PyMEs".

Se concluye que NO, la variable no es significativa y su impacto sobre el proceso de gestión de la innovación es negativo ya que resultó con beta negativa. Esto se da posiblemente porque en el caso de la industria de T.I., en la mayoría de los casos, cuando un cliente esta utilizando un producto se le hace muy pronto cuando se libera una siguiente versión y no alcanza a discernir y obtener el mejor provecho posible el consumidor cuando ya anda 
requiriendo del cambio de versión o tecnología. Para estos casos particulares los clientes y/o consumidores no son tomados en cuenta en sus necesidades ya que en algunas ocasiones se adelantan a la necesidad real y tratan de crear una necesidad de mercado que todavía no existe.

Hipótesis \# 2: "La Creatividad Organizacional impacta positivamente en el proceso de gestión de la innovación en el desarrollo de nuevos productos para PyMEs".

Se concluye que NO, ya que la significancia de esta variable esta ligeramente por encima del $5 \%$. Cabe señalar que las prácticas de esta industria es el enfocarse a adaptar el producto al cliente, sin realizar sesiones internas para generar creatividad sobre el nuevo producto. Estas empresas no suelen dedicar tiempo para la generación de creatividad tanto internamente como externamente a la organización. No ven a la creatividad como un proceso que se puede medir o sistematizar. No se visualiza a esta variable como un apoyo en la resolución de problemáticas. Se considera a la creatividad como una actividad natural del empleado, sin tomar en cuenta los extensos estudios relacionados sobre los procesos de generación de creatividad.

Hipótesis \# 3: "La Investigación \& Desarrollo impacta positivamente en el proceso de gestión de la innovación en el desarrollo de nuevos productos para PyMEs".

Se concluye que SI, ya que resultó con beta positiva y además fue la única variable con significancia menor a $5 \%$. Esta variable es la única significativa de todas y es porque en el caso de la industria de T.I. tienen muy mentalizados que este proceso es el único con el cual ellos cuentan para innovar en sus productos. Las empresas señalan que siguen explorando las nuevas tecnologías que se producen en el mercado para esta industria en particular. Se tiene un paradigma que para la solución de problemáticas esta variable es el camino para el éxito, y ciertamente hay otros procesos en los cuales se puede innovar en el largo del camino de la introducción de un nuevo producto al mercado. Los mismos apoyos del gobierno de México hacia las PyMEs hablan de los deptos. de I\&D como los pilares de la creación de innovación. Ciertamente la escuela reconstruccionista rompe con estos paradigmas. 
Hipótesis \# 4: "El Diseño de Producto impacta positivamente en el proceso de gestión de la innovación en el desarrollo de nuevos productos para PyMEs".

Se concluye que NO, ya que salió no significativa y resultó con beta positiva. Se deduce que esta industria en particular no le da valor tanto cuantitativo ni como cualitativo al diseño de producto. En el caso particular de las T.I. no se considera necesario la estética o imagen del producto ya que no es un requisito para que el consumidor compre el producto. Hay que tomar en cuenta que el diseño también abarca la funcionalidad práctica del mismo, el cómo se desempeña el producto en sus especificaciones de uso. El impacto salió positivo ya que entre más se le haya invertido en este punto se les eleva el precio del mismo y sabiendo que no existe un valor agregado lo califican como no significativo para el proceso de gestión de la innovación.

Hipótesis \# 5: "La Eficiencia Operacional impacta positivamente en el proceso de gestión de la innovación en el desarrollo de nuevos productos para PyMEs".

Se concluye que NO, ya que salió no significativa y resultó con beta positiva. Se deduce que las empresas no consideran la eficiencia operacional como de ayuda, ya que por los procesos internos de desarrollo de producto no es necesario estar en la vanguardia en las nuevas tecnologías de producción 0 administrativas. Los proyectos son realizados por pocas personas y no es necesario un complejo proceso de fabricación. El impacto salió positivo ya que entre más se le invierta no deja un valor agregado real y por eso su impacto para el proceso de gestión de la innovación no es tan relevante.

Hipótesis \# 6: "La Eficiencia Comercial impacta positivamente en el proceso de gestión de la innovación en el desarrollo de nuevos productos para PyMEs".

Se concluye que NO, ya que resultó, por mucho, no ser significativa. Esto se deduce que en el caso de la industria de T.I. la mayoría de los casos no centran estrategias de ventas por el giro del negocio, que como es un producto normalmente hecho a medida no se le invierte en publicidad. En este punto su mejor estrategia comercial es el que se pase la voz entre cliente y cliente, por no ser productos de alta rotación. 
Hipótesis general: "El proceso de gestión de la innovación para el desarrollo de nuevos productos en el sector PyMEs para el estudio al cluster de T.I. en Nuevo León es impactado positivamente por el orientación al mercado, creatividad organizacional, investigación \& desarrollo, diseño de producto, eficiencia operacional y eficiencia comercial."

Se concluye NO (para algunas variables) y SI (para una variable), ya que hay una variable impacta positivamente al modelo propuesto (investigación \& desarrollo) y las cinco restantes no son significantes y esto se deduce que es por el tipo de industria tan particular que es el de tecnologías de la información. Su dinámica es muy distinta como por ejemplo a la industria como la de consumo donde hay mucha rotación de producto y normalmente en ciertos productos la imagen del mismo es decisiva para su compra. En esta industria la rotación del producto es baja, y cada producto es hecho a la medida de la necesidad de ese cliente en particular. Aún y cuando hay productos de línea, estos resultan ser modificados al giro de negocio del cliente por sus procesos productivos, por lo que el tema de innovación no se considera una estrategia de negocio para la mayoría de las PyMEs de este sector productivo. Los cambios tan rápidos que contrae esta industria son en algunas ocasiones hasta inapropiados para algunos consumidores porque no hay tiempo para adaptarse al actual y en ocasiones no se obtiene el mejor provecho del mismo producto.

\section{Recomendaciones de la investigación}

En base a toda la información teórica y práctica que muestra este trabajo de investigación, una recomendación amplia es que se debe de apostar a los procesos formales de generación de creatividad en las organizaciones. Ya que se considera que se tiene una percepción errónea sobre el término de la creatividad, como si fuera un proceso abstracto que no se puede controlar y que solo algunos elegidos son los que generan grandes ideas revolucionarias en este mundo. La creatividad es un proceso que se puede medir y gestionar, es un proceso que se debe de considerar como uno más en la organización como por ejemplo lo es el proceso de compras, ventas, entre otros. Se debe tomar en cuenta que los beneficios que puede generar a una organización un proceso formal de generación de creatividad son mucho muy altos contra lo que se invierte en ejecutarlos. 
Ciertamente existe la necesidad de vincular aún más a la academia con la empresa, las firmas de renombre cuentan con su propia área de investigación \& desarrollo. Las PyMEs por el contrario, deben de buscar algo similar en las aulas de laboratorio de las universidades. Con esto se sigue viendo la necesidad de que gobierno, academia y las PyMEs deben caminar juntos para lograr un mayor éxito. Se necesitan empresarios y emprendedores en México que le apuesten a la Innovación, que vayan en busca de esos nichos de mercado que no han sido explorados hasta el momento y que sus ideas de nuevos negocios no busquen competir por querer una porción del mercado que ya está repartido, si no que tengan en la mira los mercados nuevos, los que no han sido explorados hasta el momento.

\section{Futuras líneas de investigación}

Este estudio mostró que cinco variables del modelo propuesto fueron rechazadas para el sector de Tecnologías de Información de Nuevo León, posiblemente debido a las características del sector analizado. Una futura línea sería aplicar el mismo instrumento en otro sector de actividad de las PYMES en Nuevo León, para ver el impacto de las seis variables del modelo propuesto hacia esa industria.

Otra línea importante, se propone como una investigación futura incluir el concepto de Liderazgo como una variable más al modelo propuesto, por ser éste una de las principales habilidades que debe de tener el dueño de la PyME para darle el empuje y la rapidez suficiente para que cada unos de los proyectos salga en tiempo, en forma y que el producto denote ser creativo e innovador. El proceso de gestión de la innovación en las organizaciones debe de venir en cascada desde la alta gerencia hasta los últimos puestos de trabajo de la organización para que se transforme paulatinamente en una cultura de trabajo.

\section{Referencias}

Amabile, T. 1995, 23 de Enero. Creativity Killers [Stevens, Tim]. Industry Week (US), sec. ISSN/ISBN 00390895, p. 63.

Amabile, T. 1998. A model of creativity and innovation in organizations. Research in 
Organizacional Behiavor. Vol.10 pp. 123-167.

APA. 2002. Manual de estilo de publicaciones de la Américan Psychological Association (Adaptado para el español). México D.F: Editorial Manual Moderno 2a EdiciónTraducida de la 5a Edición en Inglés.

Arraut, L. 2007. Elementos Clave para generar la capacidad emprendedora para el desarrollo de las organizaciones innovadoras. Recuperado el 02 de Noviembre del 2007, de http://ihm.ccadet.unam.mx/virtualeduca2007/pdf/179-LAC.pdf (producto del proyecto de investigación doctoral).

Castellanos, C. 2006. Innovación Modelo de Crecimiento. El Economista. Recuperado el 02 Noviembre del 2007, en 4inMex.

Creswell, J. W. 2003. Research Design: Qualitative, quantitative, and mixed method approaches. 2nd. Edition. Thousand Oaks: Sage Publications.

De Bono, E. 1994. El pensamiento creativo. México D.F: Paidos Plural-Titulo Original Serious Creativity. Using the Power of Lateral Thinking to Create New Ideas.

European Commission Eurostat. 1992. Oslo Manual. Europa: 1era Edición.

Fernández, E. 1996. Innovación, Tecnología y Alianzas Estratégicas. Madrid: Editorial Civitas

Forrest, J. E. 1991. Models of the Process of Technological Innovation. Technology Analysis \& Strategic Management, vol. 3, $n^{\circ}$ 4, pp. 439-453.

Hammel, G. 2002, Dec. Innovation now! (It's the only way to win today). Fast Company, 65,114-123.

Kim, W. C. \&. M. 2005. La estrategia del océano azul. Boston M.A US.: Harvard Business School Press.13.

Kim, W. C. \&. M. 2005, Feb. Navigating Toward Blue Oceans. Optimize, pp. 44-52.

Kline, S. y Rosenberg, N. 1986. An Overview of Innovation, in the Positive Sum Strategy: Harnessing Technology for Economic Growth. Washington, D.C., National Academy Press, pp. 275-305.

OECD. 2002. Reviewing the ICT sector definition: Issues for discussion. Working party on indicators for the information society. Stockholm, 25-26 April 2002.

Peralta, F. 1998. Estudio de tres modelos de creatividad: criterios para la identificación de la producción creativa. Departamento de Educación en la Universidad de Navarra. Recuperado el 09 Enero del 2010, de: http://revistas.ucm.es/edu/11368136/articulos/FAIS9898110067A.PDF

Peñaloza, M. 2002. La Industria de Software, una oportunidad para México. Año 1, Número 5, Enero de 2002.

PYME Pequeña y Mediana Empresa. 2007, Noviembre. Disponible en: http://www.pyme.com.mx

Rodríguez, M. 2001, Noviembre 01. ¡A Innovar!, las PyMEs están en una posición ideal para innovar. Contacto de Unión Empresarial. Recuperado el 02 Noviembre del 2007, en 4 inMex.

Rojas, M. \& Merchant, H. \& Ortiz, Joaquín. 2006, Nov. Conocimiento e Innovación en México: hacia una política de estado. Elementos para el Plan Nacional de Desarrollo y el Programa de Gobierno 2006-2012. Recuperado el 29 de Enero de 2008, de: http://www.foroconsultivo.org.mx/libros_editados/conocimiento_innovacion.pdf 
Rothwell, R. 1994. Towards the fifth-generation innovation process. International Marketing Review, vol. 11, n 1. pp. 7-31.

Ruelas Gossi, A. 2004, Feb. Innovar en Mercados Emergentes. Harvard Business Review, pp. 62- 71.

Takeuchi, H. y Nonaka, I. 1986. The new product development game. Stop running the relay race and take up rugby. Harvard Business Review, enero-febrero, pp. 137-146.

Velasco, E., Zamanillo, I., \& Gurutze, M. (nd). Universidad de La Rioja. Recuperado el 10 de Marzo de 2008, de:

http://dialnet.unirioja.es/servlet/fichero_articulo?codigo=2499438\&orden=0 